\title{
EFFICIENT REGISTRATION OF LAND USING BLOCK CHAIN TECHNOLOGY
}

\author{
A.Ponmalar, M.Poovarasan, T.Vasumathi, G.M.Keerthana, A.Yogesh, Dr V.Dhanakoti
}

\begin{abstract}
A Block chain as a technical solution for real estate(land registry)transactions that is a technical demo shows technology and development of the solution. In the proposed system, the knowledge about the block chain and what are all the ways the concepts of block chain will be used in real life applications such as land registry, vehicle registration, financial records,etc. In this paper we can detailed study about the land registry concept .There merits and demerits on using the concepts of block chain on this fields are also analyzed.
\end{abstract}

\section{Introduction}

What is block chain? Block chain concept was introduced by Satoshi Nakamoto in 2008 it can serve as a public ledger using its own unit of account (Bit coin)[1]. Development of a new information technology(IT) can bring change in the society. Blockchain is a technology to serve as public ledger using its own unit of account (bit coin).The invention of bit coin using blockchain concept is the first digital currency. This block chain technology involves creating a new methodology digital verification records of files. E.g. transactions[2]. This verified records are considered as fingerprints. This records are groups into blocks and they are linked together. It is generally defined as the block of chains, each and every block contains a cryptographic hash value of the previous block in the chain.

A block chain is a public or distributed ledger that is used for managing the transactions in an efficient manner. A block chain uses only a peer to peer network as a protocol for communicating all the new blocks. The third parties can't able to alter the data in any given block when the transactions

completed. But the data can be alter by doing alterations of all the subsequent blocks[3]. Data stored in a block chain are impossible to alter, rewrite, delete or do any illegal manipulation activities, it's highly secure and reliable network. Block chain is consider as the decentralized system which uses peer -to -peer network system, so there is no centralized government or organizations to control these block chain because it is a public digital ledger that is used for providing security as well as managing all the transactions

Revised Manuscript Received on July 05, 2019.

A.Ponmalar M.E,(Ph.D) Assistant Professor in Sri Sairam Institute of Technology.

Dr.V.Dhanakoti Ph.D , Associate Professor in Valliammai Engineering College.

Poovarasan, Sri Sairam Institute of Technology

Keerthana, Sri Sairam Institute of Technology

Vasumathi, Sri Sairam Institute of Technology

A.Yogesh, Sri Sairam Engineering College. across the world who are all connected to block chain so that a data cannot be altered in blocks, without the alteration of all subsequent(previous and after) blocks.

There are three types of networks available in block chain, they are public block chain, private block chain and consortium block chain.

In Public block chain there is no restrictions for accessing the data. Everybody can send a transactions through the internet connection and become a valuator. The well-known public block chains are Bit coin and Ethereum.

A private block chain is authorized as a middle-ground for companies small group's that are generally interested in the block chain technology but they are not with any level of control. Only the network administrator can allow the persons to join on block chain. There is some restrictions to access the data . This type of block chains can be considered as a private network.

A consortium block chain is a semi decentralized system. It is also authorized but instead of a single organization a unique group controlling it, a number of companies, where each might operate a node with the help of a network. In consortium chain, administrators restrict the users reading and also to see the block, this type of blockchain can allows only the restricted user that the people whom are they trust can only add them in the block and made a control with them itself.

\section{Related works}

Now a days blockchain technology is one of the most developing technology in the world, there are lot of researchers are involve they are trying to develop the technology ,let us see what are the related works done in the field. The most important aspect of blockchain concept is the security every one can easily trust the concept, first the concept was introduced for the transaction of the bit coin which is called as the online currency there is no dependency of the third party these are all done by the blockchain concept.

E-voting system is the another developing technology on the blockchain, by introducing the digital concept in voting system majority of the malpractices are reduced and this leads to the correct democracy nation if the concept are introduced by our government. The technology are also used in the financial services that are also helps in the development of 
the nation the country development is depend on the financial growth that are been maintained by the technology.

Bit coin is the online currency that can leads to lack of third party that can leads to development, at the same time it can also leads to illegal activities are done by the technology also so it is consider as the double edged sward. The data stored in the blockchain concept are can't be changed, rewrite or delete the values in the block so that is act as the public ledger and used to store the values .

That have both private and public concepts in the blockchain technology so if any growing company want to make a community circle within the limit .The maximum security level are given by the system.

The concept of blockchain is used in the various fields that is not a drop of water that is like a ocean that contains a number of technology with in this. Not only in the above mentioned fields the blockchain concept are also used in land registry.

\section{Blockchain}

\subsection{The Land Registry Today.}

The normal land registry system used in our country is the basic documentary system that is the basic and traditional process followed in our country. The information's are stored in a normal land registry system involves missing of lot of documents and it takes several time to update may causes more time delay. Now it is digitalize but not completely where you can follow the normal manual process. The following requirements are needed for land registry[5].

The old documents of the land and the owner and buyer want to appear on the registration office and put there signature in the new document in front of the registor. The patta for the land must be transferred, pan card must be compulsory for the money transaction, both members should submit there id proof in the register office and important thing is the witness of the land transaction should also sign in the new document. this can be removed by the digital process. The normal pen and paper system was followed earlier but now it is digitalized by the computer system. The major drawback in the system is the reliability that is anyone can easily been cheated by this system. In our country there are lot of illegal land registry are done and people get easily collapsed by the manual system. Many documents for land are missed which is impossible to find the real owner of the land is the major drawback for the system. Not only the missing documents there might occur some natural disaster like earthquake, landslides which will lead to the loss of the original documents of the land and there is no way to find the real owner of the land or whether the land is a private property or a public government property, At the time we may lose our digital data also ifthere is no backups are available.

\subsection{Block chain in land registry .}

As we know block chain is a decentralized network used a public ledger method as we can't modify any type of data in the block chain this technology may help in the land registry system, by the people from other countries can easily trust the system because in block chain we can't make any changes once the process was completed[6]. Block chain was first linked to the crypto currency Bit coin, but it is a amazing technology to rise up the life of million of people in a countless manner[7].

We believe it will make a large impact in the developing world, and also helping in uplift the life of the poor and the marginal people, helps in fighting corruption... and so much more[5].

So, what are all the issues with in land registries and why do we need to use the block chain to solve its issues? If any devastating earthquake happen in our country, were many countries and organizations did their best to reconstruct the nation, a major obstacle kept cropping up... there were thousands and thousands of plots of land. The correct owner could not be identified for that plots, and the instances ownerships were not be identified[8].

These issues had a major impact on recovery efforts done on this day. The constructions projects were stalled, while government and contractors wants to wait for real ownership issues to the land. The current land registry system is engaged with corruption and inefficiencies, and it leads to the unprotected citizens of our country that are suffering the most.

In many of the cities across the developing country , they are suffer from same problems regarding land registries. Many peoples doesnot haveenough confidence in the block chain system. Some are in confuse, if they legally owned a piece of land or if they have a legitimate(legal) sale process. Others citizens who are want to buy a new piece of land are not in sure about if the owner before legally owns it. Where the paper records are fully destroyed because of the disaster, this would be avoided with the introduction of block chain.

With block chain, we have a chance to find solution for many of these problems. The solution that we are bringing can give many of the key benefits of the upcoming technology, such as: an not changeable history of transactional records, so no one can ever doubt about the authenticity; all the records are permanently linked with the block system so no one can ever change a record of their own; and these records can be seen by any party, at any time. It is powerful, reliable and validating.

For the proof of concept, we're leveraging the inherent benefits of the Ethereum block chain on a smart contracts. This will create a single source for the owner and the documents of the land was kept the original user only not others. The buyer will be sure that the land being bought is the correct plot, and that the seller is clear with the owner, reducing the troubles, as well as the time and costs involved, for any given transaction.

So how does the proof of these concept work? At a high level, it will permanently record each captured transaction throughout the sale of a property. This means you can achieve near real-time state of property, traceability and transparency.

For example, a buyer and seller are wish to register their sale deed with the local authorities, who have negotiated the sale of a house and they would proceed to the government

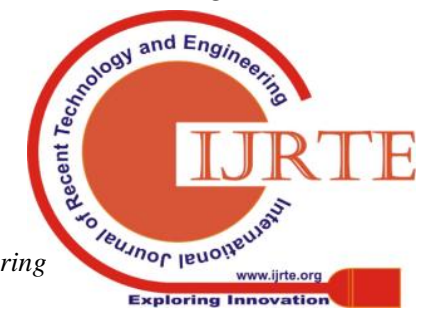


services officers as they normally would to register the sale deed, which they have in their possession.

The benefit of this system is that people involved in buying and selling property will neither require any block chain accounts or will they experience a change in the way they currently interact with the land registry system . The block chain engine simply works but it is really powerfuly in the background users . The solution will also increase citizens' confidence in the government and made reliability gain increases towards the government .The most important thing is that data security and reliability to the user.

\section{3..Hashing Technique.}

Hashing is a technique that is used for converting an user details input into cryptographic output. "Finger print" algorithm is used in hashing to receive a verification code for particular data.That code is called hash value.SHA256 algorithm is used to create a hash value.This hash value gives the digital form of an input. The working of SHA256 algorithm is by taking the third digit in a block and multiply with 7 .We can get some value, divide that value by fourth digit in a block. Combine the values in the block but not using previous calculation values .

Finally, a hash is created. For example, by using the same digital information with same hashing algorithms, the same hash will be optained.So, the same fingerprint is not used.It is unique and the hash is also unique for every single block. When we are looking at fingerprint, the person does not known what that fingerprint says and also the hash value of all digital blocks.

The details of realestate transaction present in the blocks are scanned and converted into digital information. The hash value for each transaction is unique. For example, purchasing contract is sent to bank via email, the bank can check that the contract details is correct or not. The bank then takes the information of transaction and run the details by using algorithm SHA256. The bank can receive some hash value and compare that hash with hash which is present on the verification records.

Finally, the bank can0trust the transaction is correct, is the original purchasing contract. If anybody sends an incorrect transaction contract, the hash value does not match. There is no security, so the bank can feel that is an incorrect document. Then that would became as fraud able documents, because the hashes for every documents are saved in the block chain. The block chain is a process to save the every hashes as a group . A block is a certain space to save the hashes for each transaction.

The block chain is a chained technique because each block connected back to the previous block. Each block gets a hash value which is connected to previous block, i.e. a verification, of the previous block, which makes the person as difficult to cheat by creating another block. For example, there is no possibility to enter a new verification code into an old block without changing the entire blocks.

The people can use the blockchain technique to see that changes and come to know that blockchain is not correct. A block header is the unique identity of a particular block on a block chain and is hashed by miners for rewards. Solving the hash is definitely requires a miner.

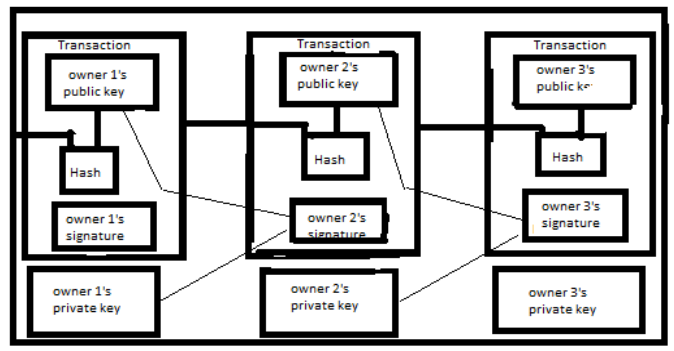

Merkle Tree

Merkle trees are a part of Blockchain Technology .A merkle tree is a structure that allows a security for the data which is stored in blockchain. This structure helps verify the content of the entire data. Merkle trees are used by both Bitcoin and Ethereum

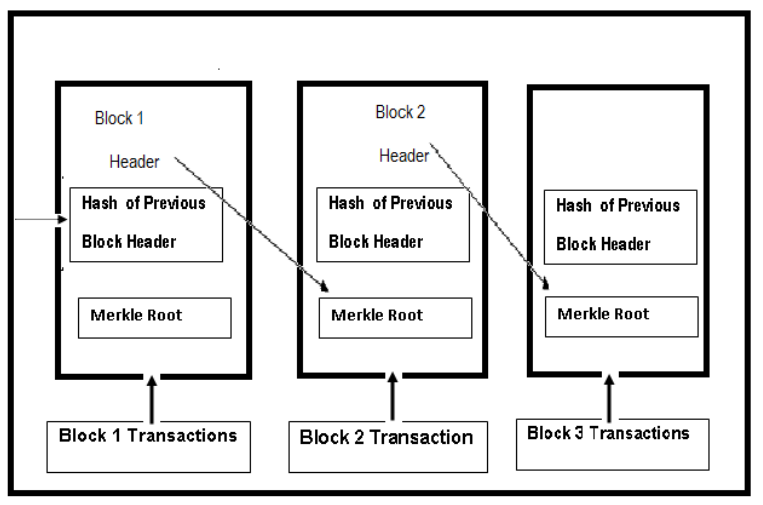

\section{How do Merkle trees works?}

A Merkle tree is used for producing a digital fingerprint of the entire set of transactions in the block, thereby enabling a user to verify. Merkle trees are created by hashing pairs until there is only one hash left (this hash is called the Root Hash, or the Merkle Root). They are constructed from the bottom up, from hashes of individual transactions (known as Transaction IDs).

Each leaf node is a hash of transactional data, and each non-leaf node is hash of previous data. Merkle trees are binary and therefore require an even number of leaf nodes. If the number of transactions is odd, the last hash will be duplicated once to create an even number of leaf nodes. Let's look at an example of four transactions in below block: A, B, C, and D. Each of these is hashed, and the hash stored in each leaf node, resulting in Hash A, B, C, and D. Consecutive pairs of leaf nodes are then hashed in a parent node.

Hash A and Hash B, hashed into Hash AB, and separately hashing Hash $\mathrm{C}$ and Hash D, are hashed in Hash CD. The two hashes (Hash $\mathrm{AB}$ and Hash $\mathrm{CD}$ ) are then hashed again to produce the Root Hash (the Merkle Root). Blocks can be hashed until there is only one node at the top. Using a Merkle tree can reduce the amount of data. It separates the valid data from the data itself. A Merkle

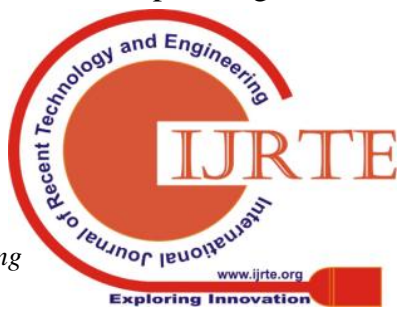


tree can work on a distributed system.

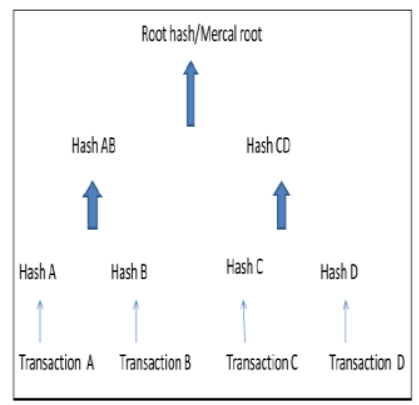

\subsection{Hyper ledger concept.[11,12,13,14]}

We can cross many type of concepts like block chain, Ethereum, Hyper ledger .In this concept of land registry we use the concept of Hyper ledger why we can't use the Ehereum? The answer is that where Ethereum is the public ledger in this block chain anyone can add blocks at the time most of them can able to know your details there is a risk in that most of them are in the same block .

So we use the Hyper ledger concept in the land registry process. this a type of public block chain in this chain we can added the persons to whom we want to add and make our boundary in a certain limit by adding a extra block in the chain that can take more verifications by the technology called hashing concept is used to add the block the way of certain verification process.

Let us see what is a Hyper ledger, first see Hyper ledger is not a company, crypto curency or even a block chain . Simply say that Hyper ledger works on basic of centralized hub technology. Where as Hyper ledger is an open source collaborative effect created to cross-industry block chain technology used in Finance, IOT, Super chain , manufacturing industries and also in developing technologies.

The main goals for Hyper ledger is to create a enterprise grade , open source, distributed ledger framework and that are in code based, provide a neural ,open and community-driven infrastructure. Build a technical communication, educate the public to aware of these technology and to promote our community of communities Now see that the frameworks that are used in the Hyper ledger are *Hyper ledger Burrow * Hyper ledger Fabric* Hyperledger indy * Hyperledger iroha *Hyperledger saw tooth they all are the framework that are commonly seen, the land registry concept is based on the Hyper ledger fabric come let us discuss about it. Hyper ledger fabric is a developing application or a solution with modular architecture that contains a block of codes that are called as the container technology called "chain code". Now the Hyper ledger fabric is the modular, scalable, secure foundation for industrial block chain solutions, these concepts are highly reliable and secure.

let's consider the person $\mathrm{A}$ and the person $\mathrm{B}$, the person $\mathrm{A}$ wants to give some amount to the person $\mathrm{B}$ that are monitored by the third party that can be declared by the introduction of block chain in the technology also have the lot of backlogs like in public block chain technology if A gives amount to B means the identity of the person $\mathrm{A}$ and $\mathrm{B}$ are known by all the persons connected with in the block chain, that is a major drawback to us, this can be eliminated by the use of Hyper ledger fabric.

In this technology the fabric connects the persons as a peer to peer that third party receive only the required amount of information that want to transfer and not know the complete information of the persons who want and send the amount, let us apply the concept in land registry system,

Person A decides to sell his product to B ,first A wants to know the query for know the B's address and the membership service works the app looks for the membership services and then verifies the B's membership now the Hyper ledger fabric connects both the parties A and B with the contract and the verification process are held on process if both parties generate a result which are then sent to the consensus cloud to be ordered and verified if the person $\mathrm{A}$ is willing to give the land to the person $\mathrm{B}$ and the person $\mathrm{B}$ accepts that he can receive the land from $A$,if both are giving the result of verification process .once the consensus cloud validates the transaction and received the product, same transactions are recorded in the ledger ... now see the benefits of Hyper ledger fabric are decentralized network, safe transactions, pluggable architecture, and that can be easily programmable and this technology are used in many companies.

\subsection{Blockchainconcept}

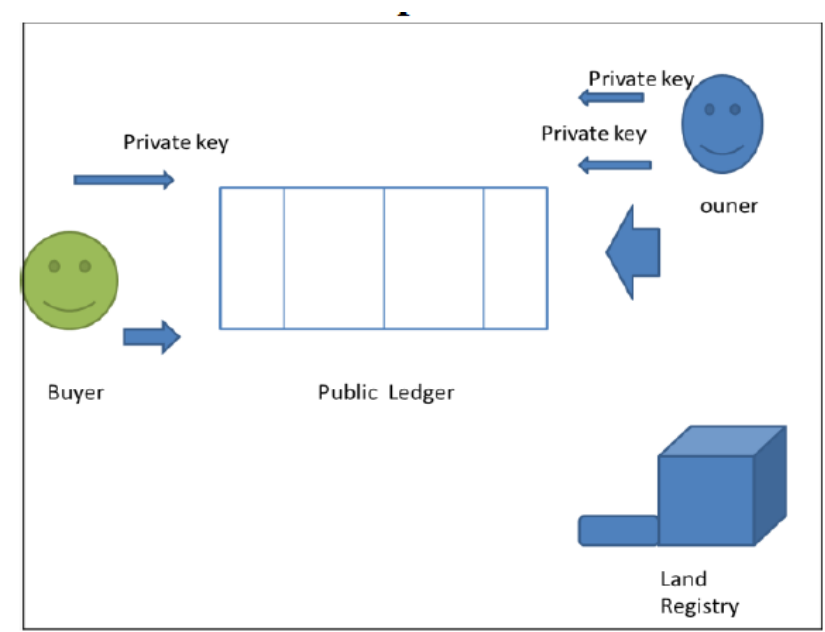

When property transactions are done by block chain there is no longer to trust the opposite party e.g. suppose if someone else went cheating that a certain coin conforms the ownership of the house. He can demonstrate that he is the real owner of an unspent coin by signing a message using his private key, and he can demonstrate the transaction history involving that coin,.

As mentioned above block chain is a public ledger the person who connected with the chain can able to see the details of others but can't able to change the things present in the system .if the person is the owner of the land he has the documents of the land in a digital form or a manual form in a ordinary system in the way the block chain are implemented the data are stored in a certain block the buyer connected with in the system can see the details of the land, there previous

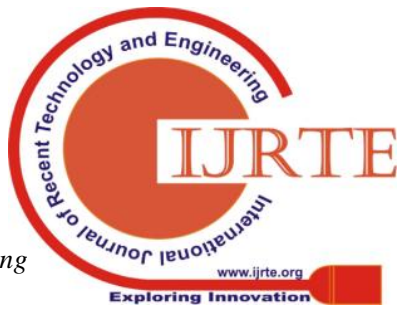


owner of the land and can see the total history of the land because the data once entered in the block chain are can 't be change or delete, we can't able to delete the data in the block chain.

If the buyer is the unknown person to the place, he does not know anyone in the place. There is a question that whom he can realize? That is solved by the block chain technology, he can easily verify that land he wants to buy is safer and reliable from in his place itself.

Let us see the block chain concept if the person is the owner of the land. He can generate two type of keys the public and the private key by the help of the public key every one can access the key and can see the value of the property and some other things that are present in the land, and generate the private key by the owner to whom want to buy this land by the private key both can exchange the amount and the documents of the land there money transaction is done by the way of bit coin ,these events are stored in a public ledger and the third person can see the land is sold for that price and brought by them with the help of block chain. The transaction data are stored in a certain block in the block chain. The third party can able to see their transaction.

\subsection{Benefits.}

By the help of block chain each transaction of land and money are become legal there is no chance to commit any illegal activities[15,16,17,18,19]. Generally in our country there is lot of malpractices done in the field of real estate, thus by introducing this concept these risks are reduced. There is no need for depending on the other authorization for this process, time saving, tension free, self satisfaction, all process are online process.

\section{Conclusion.}

Thus from this paper we can see the concepts of block chain in land registry. We have proposed the solution for the traditional way of land registry by making it digitalized using the block chain concept, for this we use the hyper ledger to provide efficient solution.

\section{5..Reference}

[1]Introduction, What is Blockchain Technology? A Step-by-Step Guide For Beginners

https://blockgeeks.com/guides/what-isblockchaintechnology/

[2]Introduction,Blockchain

https://en.wikipedia.org/wiki/Blockchain

[3]Gültekin Berahan Mermer ; Engin Zeydan ; Suayb

$\mathrm{Sb}$ Arslan,An overview of blockchain technologies:

Principles, opportunities and challenges,Published in:

2018 26th Signal Processing and Communications Applications Conference

(SIU),https://ieeexplore.ieee.org/document/84 04513

[4]Land Registry Today,AlexandruOprunencoPolicy and Innovation Specialist, UNDP in Asia and the Pacific Chami Akmeemana CEO, Block chain Learning Group and Block scale Solutionsblog/2018/Using-blockchain-tomake-

land-registry-more-reliable-in- India.html
[5]The Land Registry in the block chain ISO/TC211 Magnus Kempe, Kairos Future https://committee.iso.org/files/live/users/fh/aj/ aj/tc211 contributor\%40iso.org/files/ISO\%20 TC\%20211\%2044th\%20meeting_Wednesday $\% 20$ seminar\%20presentations/Blockchain\%2 0for\%20real\%20estate\%20and\%20other\%20 use $\% 20$ cases_Magnus\%20Kempe.pdf

[6]How cryptographic algorithms and hashing keep blockchain secure

https://jaxenter.com/cryptographic-hashingsecure-

blockchain-149464.html

[7]Blockchain concept,

Taylorwessinghttps://www.taylorwessing.co $\mathrm{m}$ /download/article-land-registrationblockchain. html

[8]Yu Wanjun ; Wu Yuan Research on Network Trading System Using Blockchain Technology,

Published in: 2018 International Conference on Intelligent Informatics and Biomedical Sciences

(ICIIBMS),

https://ieeexplore.ieee.org/docume nt/8550004

[9]Zach von Naumann The Evolution of the Cryptographic Hash Function in Blockchains https://medium.com/shokone/hash-no-notthat-

kind-the-crypto-kind-2e8bf616aa24

[10]Hashing,ASIC-Resistance of Multi-Hash Proof-of-Work Mechanisms for Blockchain Consensus ProtocolsReceived October 2, 2018, accepted October 28, 2018, date of publication October 31, 2018.

[11]Ethereum Blog. (2018). On Public and Private Block chains - Ethereum Blog. Available at:

https://blog.ethereum.org/2015/08/07/ onpublicand-private-block chains

[12]Hyperledger.Salanfe, "Setup your own private Proof-of-Authority Ethereum network with Geth", Hacker Noon, 2018. Available at:

https://tinyurl.com/ y7g362kd.

[13]Hyperledger,Getting started with Hyper ledger Composer and private blockchains

https://dev.to/damcosset/gettingstarted-

with-hyperledger-composer-andprivate-

blockchains--36ab

[14]Quoc Khanh Nguyen ; Quang Vang DangBlockchain Technology for the Advancement of the Future,Published in: 2018 4th International Conference on Green Technology and Sustainable Development(GTSD)

.https://ieeexplore.ieee.o rg/document/8595577

[15]Julija Golosova ; Andrejs Romanovs The Advantages and Disadvantages of the Blockchain Technology,

https://ieeexplore.ieee

.org/document/8592253,Published in: 2018 IEEE 6th Workshop on Advances in Information, Electronic and Electrical Engineering (AIEEE)

[16]Blockchain concept. The Land Registry in the block chain ISO/TC211 Magnus Kempe, Kairos Future

[17]Shuling Li,Application of Blockchain Technology in Smart City Infrastructure, Published in: 2018 IEEE International Conference on Smart Internet of Things (SmartIoT), 
https://ieeexplore.ieee.org/docum ent/8465562

[18]Nir Kshetri ; Jeffrey Voas,Blockchain in Developing Countries,Published in: IT Professional ( Volume:20, Issue:2 ,Mar./Apr. 2018 ),

https://ieeexplore.ieee.org/document/8 338009.

[19]https://medium.com/coinmonks/firstbusinessnetwork-using-hyperledgercomposer- ba1691c5e466 [20]Rifa Hanifatunnisa ; Budi RahardjoBlockchain based e-voting recording system design,published in: 2017

11th International Conference on Telecommunication Systems Services and Applications(TSSA),

https://ieeexplore.ieee. org/document/8272896

\section{AUTHORS PROFILE:}

A.Ponmalar M.E,(Ph.D) is working as Assistant Professor in Sri Sairam Institute of Technology.

Dr.V.Dhanakoti Ph.D is working as Associate Professor in Valliammai Engineering College.

Poovarasan,keerthana,Vasumathi are studying B.Tech in Sri Sairam Institute of Technology.

A.Yogesh is studying B.Tech in Sri Sairam Engineering College. 